\title{
A dislocation-based constitutive description for modeling the behavior of FCC metals within wide ranges of strain rate and temperature
}

\author{
J.A. Rodríguez-Martínez ${ }^{\mathrm{a}, *}$, M. Rodríguez-Millán $^{\mathrm{a}}$, A. Rusinek $^{\mathrm{b}}$, A. Arias $^{\mathrm{a}}$ \\ ${ }^{a}$ Department of Continuum Mechanics and Structural Analysis, University Carlos III of Madrid, Avda. de la Universidad 30, 28911 Leganés, Madrid, Spain \\ ${ }^{\mathrm{b}}$ National Engineering School of Metz (ENIM), Laboratory of Mechanic, Biomechanic, Polymers and Structures (LaBPS), 1 route d'Ars Laquenexy, \\ 57078 Metz Cedex 3, France
}

Keywords:

Viscoplasticity

Constitutive model

Strain rate

Thermal activation

OFHC copper

\begin{abstract}
A B S T R A C T
In this work a dislocation based constitutive description for modeling the thermo visco plastic behavior of FCC metals has been developed. The constitutive description, which is founded on the concepts of thermal activation analysis and dislocation dynamics, assumes the plastic flow additively decomposed into internal stress and effective stress. The internal stress represents the applied stress required for the transmission of plastic flow between the polycrystal grains and it is defined by the Hall Petch relationship. The effective stress formulation, which is the main innovative feature of this work, represents the thermally activated deformation behavior. This is defined taking into account the interrelationship between strain rate and temperature, and gathers structural evolution dependence. This structural evolution is described as a function of dislocations density, which acts as internal state variable in the material deformation behavior. A systematic procedure for identifica tion of the material parameters is developed and the model is applied to define the behav ior of annealed OFHC copper. The analytical predictions of the constitutive description are compared with the experimental data reported by Nemat Nasser and Li (Nemat Nasser, S., Li, Y., (1998). Flow stress of FCC polycrystals with application to OFHC Copper. Acta Mater. 46, 565 577). Good correlation between experiments and analytical predictions is found within wide ranges of strain rate and temperature.
\end{abstract}

\section{Introduction}

Over the last decades, the theoretical treatment of the constitutive behavior of metals has raised the interest of the most relevant researchers in the framework of contin uum mechanics (Austin and McDowell, 2011; Campbell, 1973; Campbell and Ferguson, 1970; Conrad, 1961; Klepaczko, 1975; Klepaczko et al., 2009; Molinari and Ravichandran, 2005; Seeger, 1957; Zaera et al., 2002; Zerilli and Armstrong, 1987). The pioneer works of Taylor (1938) and Orowan (1948) among others established the defor mation behavior of metals in terms of theory of disloca tions. Metals and alloys were considered as continuum

\footnotetext{
* Corresponding author. Tel.: +34 91624 8460; fax: +34 916249430 . E-mail address: jarmarti@ing.uc3m.es (J.A. Rodríguez-Martínez).
}

solids in which flow occurs as result of a process which in volves creation of dislocations, their motion through the crystal lattice and their annihilation or storage. Thus, strain hardening (and therefore strain softening) $\theta \quad \partial \sigma /\left.\partial \varepsilon_{p}\right|_{\varepsilon_{D T} T}$, strain rate sensitivity $m \quad \partial \sigma /\left.\partial \log \left(\dot{\varepsilon}_{p}\right)\right|_{\varepsilon_{p, T}}$ and temperature sensitivity $v \partial \sigma /\left.\partial T\right|_{\varepsilon_{p}, \varepsilon_{p}}$ of the material were directly related to dislocations' density and their velocity. Later on, the seminal investigations of Perzyna (1966), Campbell and Ferguson (1970) and Kocks et al. (1975) allowed a bet ter understanding of the mechanical behavior of polycrys talline metals. Deformation mechanisms which reside behind metals plasticity were investigated (Basinski, 1959; Conrad, 1961; Klepaczko, 1991; Klepaczko and Duffy, 1982; Kocks, 2001; Kocks and Mecking, 2003; Len non and Ramesh, 2004; Seeger, 1957; Tanner and McDo well, 1999; Taylor, 1992; Zerilli and Armstrong, 1992). 
In the wake of such works, thermal activation analysis and dislocation dynamics have been frequently applied in physically based constitutive modeling (Cai et al., 2010; Campbell and Harding, 1961; Gao and Zhang, 2010; Huang et al., 2009; Kocks et al., 1975; Nemat Nasser and Guo, 2003; Voyiadjis and Abed, 2005; Zerilli and Armstrong, 1987) of metals and alloys. Among the physically based constitutive descriptions, let us refer to those models gath ering, explicitly, structural evolution (e.g., dislocation den sity evolution) (Mecking and Kocks, 1981).

The strength of dislocation based constitutive descrip tions resides on the accurate definition of material behav iors they provide. Additionally, this type of modeling is suitable for description of so called strain rate and temper ature history effects (structure dependent) (Klepaczko, 1975; Klepaczko and Rezaig, 1996; Tanimura and Duffy, 1986). Moreover it has to be remarked that this type of constitutive description is required to understand dynamic failure mechanics which are often dislocation controlled (Campagne et al., 2005). The relationship between struc tural evolution and failure mechanisms has been recently explored in relevant publications by Rittel and co workers (Dolinski et al., 2010; Rittel et al., 2006). They suggested that formation and propagation of plastic instabilities lead ing to failure in dynamically loaded metals "are most likely related to the specific microstructure (e.g., dislocation pat terns and hardening) that results from dynamic loading only" (Rittel et al., 2006).

Thus, in this paper a dislocation based constitutive rela tion with application to FCC metals is developed. The model lies on the concept of one internal state variable (mean den sity of total dislocations), which defines the structure evolu tion during the course of plastic deformation. The formulation is based on the additive decomposition of the plastic flow into internal and effective stress. The internal stress represents the athermal material behavior which is de fined strain independent. The effective stress represents the thermally activated deformation behavior. This is defined taking into account the interrelationship between strain rate and temperature. It describes the structure evolution during the course of plastic deformation. A systematic procedure for identification of the material parameters is developed and the model is applied to define the behavior of annealed OFHC copper. The analytical predictions of the constitutive descrip tion are compared with the experimental data reported by Nemat Nasser and Li (1998). The resulting constitutive mod el is notable for its ability to define the material behavior un der wide ranges of strain rate and temperature.

\section{Formulation of the constitutive description}

The formulation of the constitutive model is founded on three well established assumptions:

- Plastic deformation in shear is the fundamental mode of deformation in metals plasticity.

- The low temperature micro mechanisms involved in dynamic plasticity operate typically within the range $0 K \leqslant T_{0} \leqslant T_{m} / 2$ where $T_{m}$ is melting temperature. Those are the temperature limits of the model.
- The overall flow stress is decomposed into two additive stress components, Eq. (1): the internal stress $\tau_{\mu}$ (ather mal stress) and the effective stress $\tau^{*}$ (thermal stress).

$$
\tau \quad \tau_{\mu}+\tau^{*}
$$

Assuming Huber Mises plasticity, Eq. (1) can be rewrit ten in form of equivalent quantities operating via the Taylor factor, Eq. (2):

$\sigma \quad \sigma_{\mu}+\sigma^{*}$

Definitions of both, equivalent internal stress $\bar{\sigma}_{\mu}$ and equivalent effective stress $\bar{\sigma}^{*}$ are given next.

\subsection{The internal stress}

For FCC metals can be assumed that the internal stress $\bar{\sigma}_{\mu}$ represents the applied stress required for the transmis sion of plastic flow between the polycrystal grains (Zerilli and Armstrong, 1987), the so called Hall Petch effect. According to several authors (Rusinek et al., 2010; Voyiadjis and Abed, 2005; Voyiadjis and Almasri, 2008) this allows giving a strain independent definition of the athermal stress $\bar{\sigma}_{\mu}$ via Eq. (3). The Hall Petch effect controls the flow stress level.

$\sigma_{\mu} \quad \alpha_{1} \cdot E(T) \cdot\left(\frac{b}{D}\right)^{1 / 2}$

where $\alpha_{1}$ is a material constant, $b$ is the Burger's vector, $D$ is the average grain size and $E(T)$ is the Young's modulus temperature dependent (Klepaczko 1998) given by Eq. (4).

$E(T) \quad E_{0}\left\{1 \quad \frac{T}{T_{m}} \exp \left[\theta *\left(\begin{array}{ll}1 & \frac{T_{m}}{T}\end{array}\right)\right]\right\} \quad T>0$

where $E_{0}, T_{m}$ and $\theta^{*}$ denote respectively the Young's mod ulus at $T=0 \mathrm{~K}$, the melting temperature and the character istic homologous temperature. In the case of FCC metals $\theta^{*} \approx 0.9$ as discussed by Rusinek et al. (2009).

It has to be noted that drag mechanisms are not consid ered in the internal stress formulation of the model here proposed. Thus, the maximum rate level for which this constitutive description shows applicability is restricted to the appearance of the drag regime. In agreement with several authors that limiting strain rate level is estimated within the range $\dot{\bar{\varepsilon}}_{p}^{\max } \approx 10^{3} s^{1} 10^{4} s^{1}$ (Huang et al., 2009; Kumar et al., 1968).

Next, the formulation of the effective stress is introduced.

\subsection{The effective stress}

For FCC metals is assumed that the rate controlling mechanism is the overcoming of dislocation forests by individual dislocations. Therefore the effective stress is ex pected to be structure (strain) dependent. In order to pro vide a suitable definition for the effective stress let us follow the procedure detailed in this section of the paper.

The free energy of activation $\Delta G\left(\sigma^{*}\right)$ is tied to strain rate by the generalized Arrhenius equation (Johnston and Gil man, 1959), Eq. (5). 
$\dot{\varepsilon}_{p} \quad \dot{\varepsilon}_{r} \cdot \exp \left(\frac{\Delta G\left(\sigma^{*}\right)}{k \cdot T}\right)$

where $\dot{\varepsilon}_{r}$ is the frequency factor (so called pre exponential factor) and $k$ is the Boltzmann constant.

Moreover, the relation between the free energy of activa tion $\Delta G\left(\sigma^{*}\right)$ and the thermally activated stress component $\sigma^{*}$ is taken from Kocks et al. (1975), Eq. (6). This phenomeno logical expression has been frequently applied in constitu tive modeling over the last decades (Gao and Zhang, 2010; Nemat Nasser and Li, 1998; Uenishi and Teodosiu, 2004).

$\Delta G\left(\sigma^{*}\right) \quad G_{0} \cdot\left[1 \quad\left(\frac{\sigma^{*}}{\hat{\sigma}^{*}}\right)^{p}\right]^{q}$

where $G_{0}$ is the free energy required to overcome the bar rier without the aid of external applied stress and $\hat{\sigma}^{*}$ is considered the threshold thermal stress which (for FCC metals) is structure dependent. Moreover, $p$ and $q$ are material constants defining the profile of the obstacle. Typ ical values reported in the literature for those constants are $1<p \leqslant 2$ and $0<q \leqslant 1$ (Uenishi and Teodosiu, 2004). In this work let us consider $q=1$ (Uenishi and Teodosiu, 2004) while $p$ will be treated as a material constant which determines instantaneous temperature and rate sensitivi ties of the material flow stress.

Combination of previous expressions Eqs. (5) and (6) leads to Eq. (7).

$\sigma^{*} \quad \hat{\sigma}^{*} \cdot\left\langle 1 \frac{k \cdot T}{G_{0}} \cdot \ln \left(\frac{\dot{\varepsilon}_{r}}{\dot{\varepsilon}_{p}}\right)\right\rangle^{1 / p}$

where the McCauley operator is defined as follows $\langle\bullet\rangle$ if $\langle\bullet\rangle \geqslant 0$ or $\langle\bullet\rangle \quad 0$ if $\langle\bullet\rangle \leqslant 0$.

For simplification, and according to Rusinek and Klep aczko (2001), from this point on let us consider the ratio $\frac{k \cdot T}{G_{0}}$ equivalent to $\frac{D_{1} \cdot T}{T_{m}}$ where $D_{1}$ is a non dimensional con stant proportional to $\ln (10)$ (coming from application of the logarithms properties) and $T_{m}$ is the melting tempera ture introduced to attain non dimensionality.

Application of this procedure leads to the generalized expression for the effective stress used in the present con stitutive model (Rusinek and Klepaczko, 2001), Eq. (8).

$\sigma^{*} \quad \hat{\sigma}^{*} \cdot\left\langle 1 \quad D_{1}\left(\frac{T}{T_{m}}\right) \cdot \log \left(\frac{\dot{\varepsilon}_{r}}{\dot{\varepsilon}_{p}}\right)\right\rangle^{1 / p}$

The next step is to particularize Eq. (8) for FCC polycrystal line metals. According to several authors (Klepaczko, 1975; Nemat Nasser and Li, 1998), in previous expression the threshold thermal stress $\hat{\sigma}^{*}$ and the frequency factor $\dot{\varepsilon}_{r}$ may take into account the strain dependence of the effec tive stress. Subsequently, an original procedure to set such dependence is provided.

In agreement with Nemat Nasser and Li (1998), the threshold thermal stress $\hat{\sigma}^{*}$ is related to $G_{0}$ by the following expression, Eq. (9).

$\hat{\sigma}^{*} \frac{M \cdot G_{0}}{V^{*}}$

where $M$ is the Taylor factor and $V^{*}$ is the activation volume which is related to the average distance the dislocations move between barriers $d$ by Eq. (10) (Voyiadjis and Abed, 2005).
$V^{*} \quad A \cdot b \quad d \cdot \frac{b^{2}}{2}$

where $A$ is the activation area and $b$ is the Burger's vector.

Moreover, according to Voyiadjis and Abed (2005), let us define $G_{0}$ by the following expression, Eq. (11).

$G_{0} \quad \alpha_{2 i} \cdot \mu(T) \cdot b^{3}$

where $\alpha_{2 i}$ is a material constant and $\mu(T)$ is the tempera ture dependent elastic shear modulus.

Combination of Eqs. (9) and (11) allows relating the thermal threshold stress $\hat{\sigma}^{*}$ to the internal structure as fol lows (Voyiadjis and Abed 2005), Eq. (12).

$\hat{\sigma}^{*} \frac{M \cdot \alpha_{2 i} \cdot \mu(T) \cdot b^{3}}{V^{*}}$

By application of Eq. (10), previous formula can be re writ ten in the following form, Eq. (13).

$\hat{\sigma}^{*} \quad \frac{\alpha_{2} \cdot E(T) \cdot b}{d}$

$\alpha_{2} \quad 2 \alpha_{2 i}$

where $E(T)=\mu(T) \quad M$ is the Young's modulus temperature dependent defined by Eq. (4).

The average distance between barriers $d$ depends on the type of the barrier. In the case of FCC metals, short range barriers (those that can be overcome with thermal aid) are assumed the dislocation forest which intersects the slip plane (Zerilli and Armstrong, 1987). In such a case ( fol lowing Nemat Nasser and Li (1998)) the average distance between barriers (dislocation forest) $d$ is the same as the average spacing $\ell$. The latter is a function of the current (mean) density of total dislocations $\rho$ (mobile and immobile dislocations over few subgrains).

According to several authors the following expression is inferred, Eq. (14). This formula approximates the average spacing to the inverse square root of the current disloca tion density (Estrin, 1998; Kocks and Mecking, 2003; Kubin and Estrin, 1990; Nemat Nasser and Li, 1998). Thus, mean density of total dislocation in a generalized form can be defined proportional (the proportionality constant will be derived later) to a dimensionless function strain and rate dependent $\Psi\left(\varepsilon_{p}, \dot{\varepsilon}_{p}\right)$ (according to experiments for $\mathbf{F C C}$ metals, this function is considered as temperature indepen dent), Eq. (14).

$d \quad \ell \approx \rho^{1 / 2} \propto \frac{1}{\Psi\left(\varepsilon_{p}, \dot{\varepsilon}_{p}\right)}$

Finally, Taylor's equation (Taylor 1938), Eq. (15), emerges substituting previous expression into Eq. (13).

$\hat{\sigma}^{*} \quad \alpha_{2} \cdot E(T) \cdot b \cdot \rho^{1 / 2}$

At this point is required to determine an expression for the accumulation process of material dislocation density as a function of plastic deformation. For that task, let us follow the procedure developed by Klepaczko (1975). The struc tural evolution at different strain rates is defined by the following expression, Eq. (16). 
$d \rho / d \varepsilon_{p} \quad M_{I I} \quad k_{a}\left(\dot{\varepsilon}_{p}\right) \cdot\left(\begin{array}{ll}\rho & \rho_{0}\end{array}\right)$

where $M_{I I}$ is the multiplication factor that may be consid ered constant (Voyiadjis and Abed 2005), $\rho_{0}$ is the initial dislocation density and $k_{a}\left(\dot{\varepsilon}_{p}\right)$ is the rate dependent annihi lation factor. The latter defined in this work by the follow ing phenomenological rate dependent formulation which simplifies that proposed by Klepaczko and Rezaig (1996) Eq. (17).

$k_{a}\left(\dot{\varepsilon}_{p}\right) \quad k_{0}\left(\frac{\dot{\varepsilon}_{p}}{\dot{\varepsilon}_{a_{0}}}\right)^{2 m_{0}}$

where $k_{0}$ is the reference annihilation factor, $\dot{\varepsilon}_{a_{0}}$ is the ref erence strain rate for annihilation assumed constant $\dot{\varepsilon}_{a_{0}} 210^{10} s^{1}$ (Nemat Nasser and $\mathrm{Li}, 1998$ ) and $m_{0}$ is the absolute rate sensitivity due to defect annihilation. Pre vious formula predicts decreasing annihilation of disloca tions as the deformation rate rises, in agreement to the experimental evidences reported in the literature (Meyers et al., 2003), Fig. 1.

Integration of Eq. (16) at constant strain rate gives the following closed expression for the dislocation density, Eq. (18).

$\rho \quad \rho_{0}+\frac{M_{I I}}{k_{a}\left(\dot{\varepsilon}_{p}\right)} \cdot\left\{1 \quad \exp \left[k_{a}\left(\dot{\varepsilon}_{p}\right) \cdot \varepsilon_{p}\right]\right\}$

Dislocation density derived from previous expression, Eq. (18) increases with strain and strain rate as follows, Fig. 2.

From Eq. (18), we define the proportionality constant $\rho_{0}{ }^{1 / 2}$ and the function $\Psi\left(\varepsilon_{p}, \dot{\varepsilon}_{p}\right)$ arises in the following form, Eq. (19). Therefore, the function $\Psi\left(\varepsilon_{p}, \dot{\varepsilon}_{p}\right)$ increases with strain and strain rate.

$$
\begin{aligned}
\Psi\left(\varepsilon_{p}, \dot{\varepsilon}_{p}\right) & \left(\frac{\rho}{\rho_{0}}\right)^{1 / 2} \\
& \left(1+\frac{M_{I I}}{\rho_{0} \cdot k_{a}\left(\dot{\varepsilon}_{p}\right)} \cdot\left\{1 \quad \exp \left[k_{a}\left(\dot{\varepsilon}_{p}\right) \cdot \varepsilon_{p}\right]\right\}\right)^{1 / 2}
\end{aligned}
$$

In addition $\Psi\left(\varepsilon_{p}, \dot{\varepsilon}_{p}\right)$ can be defined in terms of the current average spacing $\ell \approx \rho^{1 / 2}$ and the initial average spacing $\ell_{0} \approx \rho_{0}{ }^{1 / 2}$ as follows, Eq. (20).
$\Psi\left(\varepsilon_{p}, \dot{\varepsilon}_{p}\right) \quad \frac{\ell_{0}}{\ell}$

Substitution of Eq. (19) into Eq. (15) leads to the following expression, Eq. (21).

$\hat{\sigma}^{*}\left(\varepsilon_{p}, \dot{\varepsilon}_{p}, T\right) \quad \alpha_{2} \cdot E(T) \cdot b \cdot \rho_{0}^{1 / 2} \cdot \Psi\left(\varepsilon_{p}, \dot{\varepsilon}_{p}\right)$

Previous formula is inserted into Eq. (8), and the resulting expression for the effective stress $\bar{\sigma}^{*}\left(\bar{\varepsilon}_{p}, \dot{\bar{\varepsilon}}_{p}, T\right)$ reads as fol lows, Eq. (22).

$$
\begin{aligned}
\sigma^{*}\left(\varepsilon_{p}, \dot{\varepsilon}_{p}, T\right) & \alpha_{2} \cdot E(T) \cdot b \cdot \rho_{0}^{1 / 2} \cdot \Psi\left(\varepsilon_{p}, \dot{\varepsilon}_{p}\right) \\
& \cdot\left\langle 1 \quad D_{1}\left(\frac{T}{T_{m}}\right) \cdot \log \left(\frac{\dot{\varepsilon}_{r}}{\dot{\varepsilon}_{p}}\right)\right\rangle^{1 / p}
\end{aligned}
$$

Now, let us define the frequency factor $\dot{\varepsilon}_{r}$ using Orowan's equation (Orowan 1948), Eq. (23).

$\dot{\varepsilon}_{r}\left(\rho_{m}\right) \quad b \cdot d \cdot \rho_{m} \cdot \varpi$

where $\rho_{m}$ is the density of mobile dislocations and $\omega$ is the attempt frequency.

In the case FCC crystals, for dislocations as barriers, the frequency factor should be considered dependent on dislo cation density, Nemat Nasser and Li (1998). In the present case, according to Eqs. (14) (20) the frequency factor for mulation $\dot{\varepsilon}_{r}\left(\rho_{m}\right)$ may be expressed in the following man ner, Eq. (24).

$$
\begin{aligned}
& \dot{\varepsilon}_{r}\left(\rho_{m}\right) \quad \frac{\dot{\varepsilon}_{0}\left(\rho_{m}\right)}{\Psi\left(\varepsilon_{p}, \dot{\varepsilon}_{p}\right)} \\
& \dot{\varepsilon}_{0}\left(\rho_{m}\right) \quad b \cdot \ell_{0} \cdot \rho_{m} \cdot \varpi \quad \text { cte }
\end{aligned}
$$

where $\dot{\varepsilon}_{0}\left(\rho_{m}\right)$, Eq. (25), is defined as the reference (initial) frequency factor considered constant in the present formu lation, $\dot{\varepsilon}_{0}\left(\rho_{m}\right) \quad 210^{10} s^{1}$ (Nemat Nasser and Li, 1998).

It has to be noted that since one internal state variable is assumed in the present model, the mobile dislocation density $\rho_{m}$ will be treated as a fraction $f$ of the mean dislo cation density $\rho_{m}=f \quad \rho$ where $f$ will be assumed constant for simplification. Therefore, we re write Eq. (25) as follows, Eq. (26).

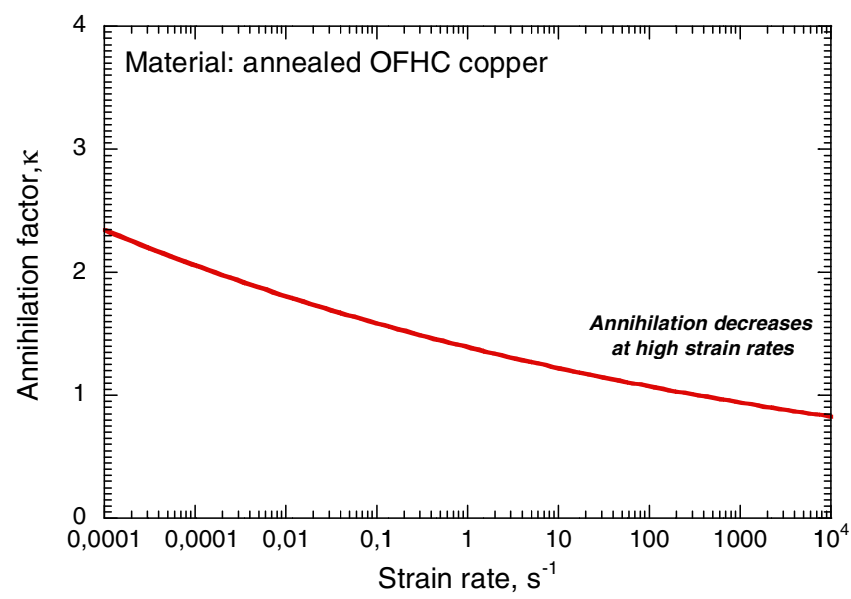

Fig. 1. Annihilation factor as a function of strain rate. $k_{0}=0.366, m_{0}=0.0282$. 


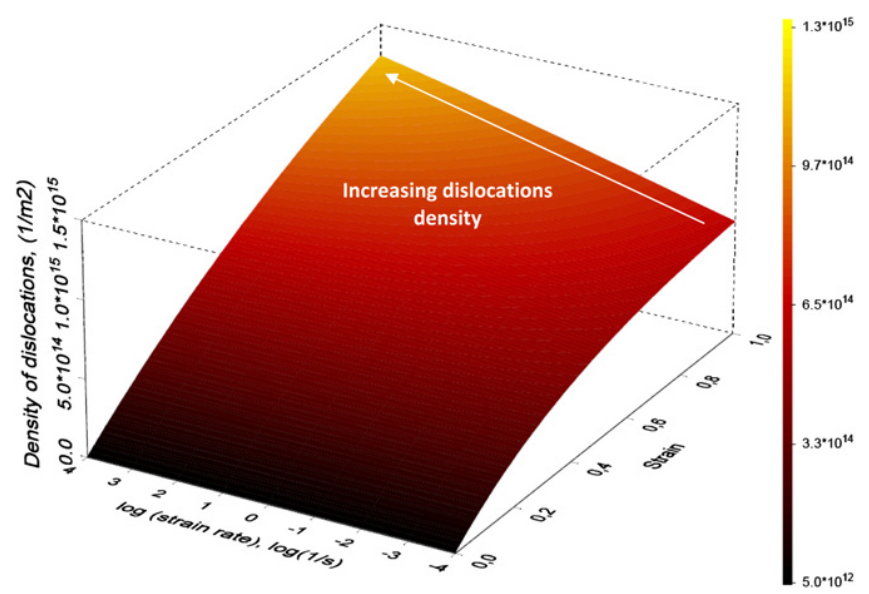

Fig. 2. Density of dislocations as a function of strain and strain rate at $T_{0}=296 \mathrm{~K} . \rho_{0}=5 * 10^{12} \mathrm{~m}^{2}, k_{0}=0.366, m_{0}=0.0282, M_{I I}=1900 * 10^{12} \mathrm{~m}{ }^{2}$.

$\dot{\varepsilon}_{0}(\rho) \quad b \cdot \ell_{0} \cdot f \cdot \rho \cdot \varpi \quad 2 \cdot 10^{10} s{ }^{1}$

Then Eq. (24) is normalized via the strain rate $\dot{\varepsilon}$ and subse quently this is expressed in logarithmic form as follows, Eq. (27).

$\log \left(\frac{\dot{\varepsilon}_{r}}{\dot{\varepsilon}_{p}}\right) \quad \log \left(\frac{\dot{\varepsilon}_{0}}{\dot{\varepsilon}_{p}}\right) \quad \log \left(\Psi\left(\varepsilon_{p}, \dot{\varepsilon}_{p}\right)\right)$

Previous formula can be inserted into Eq. (22), and the final formulation for the effective stress $\bar{\sigma}^{*}\left(\bar{\varepsilon}_{p}, \dot{\bar{\varepsilon}}_{p}, T\right)$ is obtained, Eq. (28).

$$
\begin{aligned}
\sigma^{*}\left(\varepsilon_{p}, \dot{\varepsilon}_{p}, T\right) & \alpha_{2} \cdot E(T) \cdot b \cdot \rho_{0}^{1 / 2} \cdot \Psi\left(\varepsilon_{p}, \dot{\varepsilon}_{p}\right) \\
& \cdot\left\langle\begin{array}{cc}
1 & \left.D_{1}\left(\frac{T}{T_{m}}\right) \cdot\left\langle\log \left(\frac{\dot{\varepsilon}_{0}}{\dot{\varepsilon}_{p}}\right) \log \left(\Psi\left(\varepsilon_{p}, \dot{\varepsilon}_{p}\right)\right)\right\rangle\right\rangle^{1 / p}
\end{array}\right.
\end{aligned}
$$

where the function $\Psi\left(\bar{\varepsilon}_{p}, \dot{\bar{\varepsilon}}_{p}\right)$ describes the influence of plastic strain on the rate sensitivity of the material.

In the case of adiabatic conditions of deformation the constitutive relation is combined with the energy balance principle, Eq. (29). Such relation allows for an approxima tion of the thermal softening of the material via the adia batic heating.

$\Delta T\left(\varepsilon_{p}, \sigma\right) \quad \frac{\beta}{\rho^{*} C_{p}} \int_{0}^{\varepsilon_{p}^{\max }} \sigma\left(\varepsilon_{p}, \dot{\varepsilon}_{p}, T\right) d \varepsilon_{p}$

where $\beta$ is the Taylor Quinney coefficient assumed con stant, $\rho^{*}$ is the material density and $C_{p}$ is the specific heat at constant pressure. Transition from isothermal to adia batic conditions is assumed at $\dot{\bar{\varepsilon}}_{p} 10 s^{1}$ in agreement with experimental observations and numerical estimations reported in the literature (Oussouaddi and Klepaczko, 1991; Rusinek et al., 2007).

\section{Identification and sensitivity analysis of the constitutive model parameters}

In this section of the paper, a systematic procedure for identification of the model parameters is proposed. The procedure applies the least square method to obtain the best fitting condition between experiments and analytical predictions. Then, a parametric study on the influence of material constants on the analytical predictions of the model is conducted.

\subsection{A straightforward method for identification of the material parameters}

For identification of the model parameters, three tests at different strain rates and room temperature are re quired. A test performed under quasi static loading is needed $\dot{\bar{\varepsilon}}_{p} \approx 10{ }^{3} s^{1}$ (the one taken as reference). The other two remaining tests should be at higher rate level within the range $1 s{ }^{1} \leqslant \dot{\bar{\varepsilon}}_{p} \leqslant 10^{3} s{ }^{1}$. Thus, the main steps neces sary for identification of the material constants are:

i. The first step is to identity the initial yield stress at low strain rates and room temperature, $\left.\bar{\sigma}\right|_{\varepsilon_{p}} ^{T} \quad \begin{array}{ll}293 K, \varepsilon_{p} & \\ & \end{array}$ the increase of the initial yield stress with strain rate is related to the effective stress component, Eq. (30).

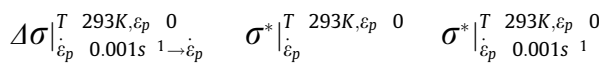

Previous relation allows for defining the value of the mate rial constants $\alpha_{2}, D_{1}$ and $p$. Thus, the contribution of the internal stress and the effective stress to the initial yield stress is determined, Eq. (31).

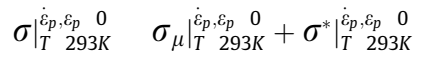

Previous expression allows for identification of the $\alpha_{1}$ value.

ii The second step is to apply the complete formula tion of the model to fit an experimental curve of the type $\left.\bar{\sigma} \quad \bar{\varepsilon}_{p}\right|_{\varepsilon_{p} \quad 0.001 s \quad 1} ^{T}$. The value of the material constant $M_{I I}$ is identified. Additionally, a reference value $\left.k_{a}\right|_{\varepsilon_{p}} ^{T} \operatorname{lo001s}_{1}$ is obtained.

iii. Again the complete formulation of the model is applied to fit three experimental curves of the type $\left.\bar{\sigma} \quad \bar{\varepsilon}_{p}\right|_{\varepsilon_{p}} ^{T}$. Then, the stress dependency upon strain rate for the function $k_{a}\left(\dot{\bar{\varepsilon}}_{p}\right)$ can be determined. This step allows identification of the material constants $k_{0}$ and $m_{0}$. 
This straightforward method for identification of the model parameters ensures the uniqueness of their values. By application of this procedure, the model has been cali brated for annealed OFHC copper using the relevant exper imental data reported by Nemat Nasser and Li (1998). The set of material constants found is given in Table 1 .

Physical constants of annealed copper are listed in Table 2. Constants on the left side of Table 2 are easily obtained from material handbooks. Constants on the right side of Table 2 come from metallurgical examinations and they can be found in the literature (Nemat Nasser and $\mathrm{Li}$, 1998; Underwood, 1970).

Next, taking as reference the values for annealed OFHC copper listed in Table 1, a parametric study on the influ ence of material constants on the analytical predictions of the model is carried out.

\subsection{Parametric study on the influence of material constants on the analytical predictions of the model}

The parametric study is split into two parts, first fitting material constant involved in the internal stress formula tion is examined, and second fitting material constants in volved in the effective stress formulation.

\subsubsection{Material constant involved in the internal stress formulation}

The fitting constant involved in the internal stress for mulation is $\alpha_{1}$, Eq. (3), which is related to the material flow stress level. The resulting model predictions are illustrated in Fig. 3. It can be observed that variations of the parameter $\alpha_{1}$ have small impact on the stress strain response of the material. This is caused by the minor role played by the internal stress on the behavior of annealed FCC metals.

Next, the influence of effective stress material parame ters on the analytical predictions of the model is examined.

\subsubsection{Material constants involved in the effective stress formulation}

First let us consider the fitting constants directly tied to the dislocation density formulation, $M_{I I}, k_{0}$ and $m_{0}$, Eqs. (17), (18). The resulting model predictions are illustrated in Fig. 4. The multiplication factor $M_{I I}$ plays a major role on the analytical predictions of the constitutive model, it controls flow stress level and strain hardening, Fig. 4a. Moreover, the constants involved in the annihilation factor formulation, $k_{0}$ and $m_{0}$, determine material strain softening at large strains, Fig. $4 \mathrm{~b}$ and c. As $k_{0}$ and $m_{0}$ increase material strain softening does also.

Next let us consider the fitting constants $\alpha_{2}$ and $p$, which are involved in the complete effective stress formu lation, Eq. (22). The resulting model predictions are illus trated in Fig. 5. The parameter $\alpha_{2}$ strongly affects material description, it controls material (strain dependent) rate sensitivity and therefore defines flow stress level and strain hardening rate, Fig. 5a. Moreover, the material parameter $\mathrm{p}$ determines material rate sensitivity; as $\mathrm{p}$ in creases flow stress level and strain hardening do also, Fig. 5b.

Next let us consider the material parameters $q$ and $\dot{\varepsilon}_{0}$, which are involved in the material rate sensitivity defini tion, Eq. (22). It has to be noticed that their value was as sumed (it was not identified in the calibration procedure) according to the considerations reported elsewhere (Ueni shi and Teodosiu, 2004; Nemat Nasser and Li, 1998).

The resulting analytical predictions of the model are de picted in Fig. 6. The parameter $q$ (in the same manner than the parameter $p$ previously analyzed) determines material ( strain dependent) rate sensitivity; as $q$ increases material flow level and strain hardening also do, Fig. 6a. Moreover, it has to be noted that the parameter $\dot{\varepsilon}_{0}$ plays a secondary role on the material description; variations of this constant have small impact on the stress strain response of the material, Fig. 6b.

In the following section of the paper, the analytical pre dictions of the constitutive model are correlated with experimental data reported by Follansbee (1986) and Ne mat Nasser and Li (1998).

\section{Application of the constitutive model to describe the behavior of annealed OFHC copper within wide ranges of strain rate and temperature}

Polycrystalline OFHC copper is a typical FCC metal that has been widely used to explore the dependence of the flow stress on strain, strain rate and temperature.

By application of the material constants listed in Tables 1 and 2, the contribution of each stress component to the overall flow stress is illustrated in Fig. 7. It is interesting to highlight that material flow stress is basically ruled by the effective stress. This is in agreement with the consider ations reported by several authors for different annealed FCC metals (Rusinek et al., 2010; Voyiadjis and Almasri, 2008).

Table 1

Value of the model constants determined for annealed OFHC copper.

\begin{tabular}{lllllllll}
\hline$D_{1}(-)$ & $p(-)$ & $\alpha_{1}(-)$ & $k_{0}(-)$ & $M_{I I}\left(\mathrm{~m}^{2}\right)$ & $m_{0}(-)$ & $\alpha_{2}(-)$ & $\varepsilon_{0}\left(\mathrm{~s}{ }^{1}\right)$ & $\varepsilon_{a_{0}}\left(\mathrm{~s}{ }^{1}\right)$ \\
\hline 0.134 & 1.1 & 0.0281 & 0.366 & $1.9 * 10^{15}$ & 0.0282 & 0.607 & $2 * 10^{10}$ \\
\hline
\end{tabular}

Table 2

Physical constants for annealed OFHC copper (Nemat-Nasser and Li, 1998; Underwood, 1970).

\begin{tabular}{lllllll}
\hline$E_{0}(\mathrm{GPa})$ & $C_{p}\left(\mathrm{Jkg}{ }^{1} \mathrm{~K}^{1}\right)$ & $\beta(-)$ & $\rho\left(\mathrm{kgm}^{3}\right)$ & $\mathrm{T}_{\mathrm{m}}(\mathrm{K})$ & $b(m)$ & $\rho_{0}\left(m^{2}\right)$ \\
\hline 130 & 385 & 0.9 & 8960 & 1340 & $2.56 * 10^{10}$ & $5.0 * 10^{12}$ \\
\hline
\end{tabular}




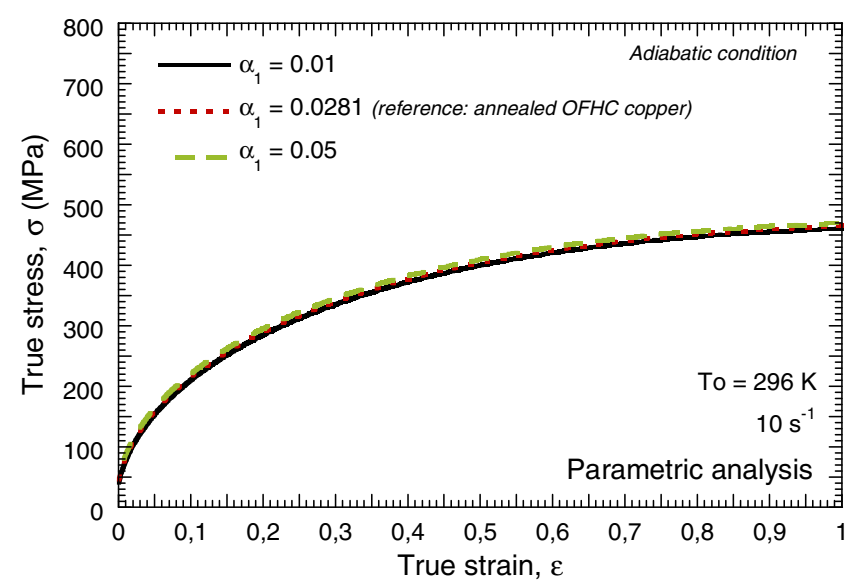

Fig. 3. Flow stress as a function of strain for different values of the material parameter $\alpha_{1}$. Loading conditions: $10 \mathrm{~s}^{1}$ and $T_{0}=296 \mathrm{~K}$.
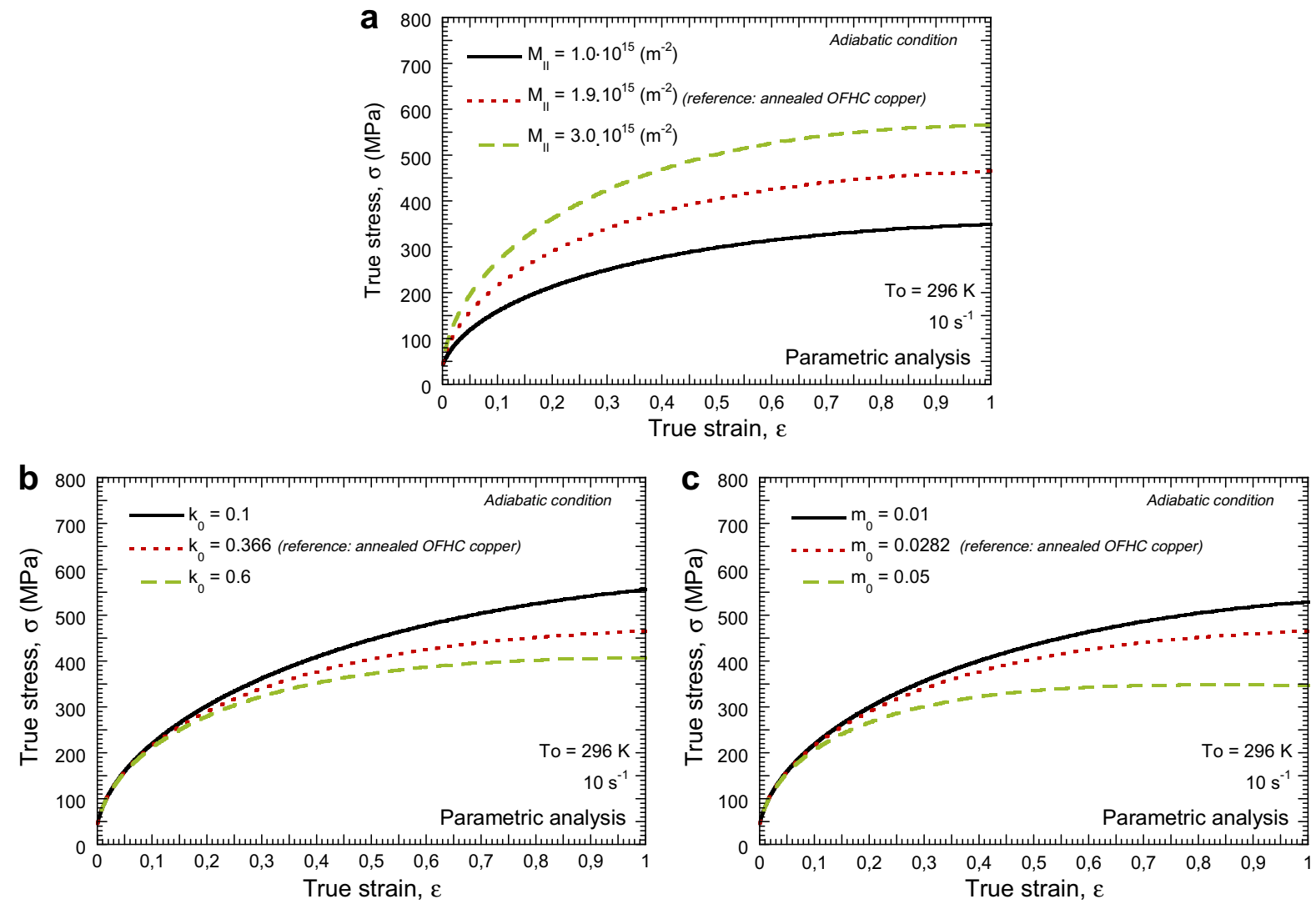

Fig. 4. Flow stress as a function of strain for different values of the material parameters (a) $M_{I I}$, (b) $k_{0}$ and (c) $m_{0}$. Loading conditions: $10 \mathrm{~s}{ }^{1}$ and $T_{0}=296 \mathrm{~K}$.

Next, the model predictions are correlated with experi ments for different strain rate levels at room temperature, Fig. 8. The constitutive description is revealed suitable for defining the flow stress as well as the strain hardening rate of the material for the loading conditions considered (the induced error is in any case less than 10\%).

It is observed that the strain hardening of the material is much stronger affected by the loading rate than the initial yield stress (due to the minor role played by the Peierls stress on the deformation behavior of most annealed FCC metals). This typical behavior of polycrystalline FCC metals is illus trated in Fig. 9, where the analytical predictions of the material flow stress evolution are represented as a function of strain and strain rate.

In other words, the material rate sensitivity (at constant strain) is dependent on the plastic strain as illustrated in 

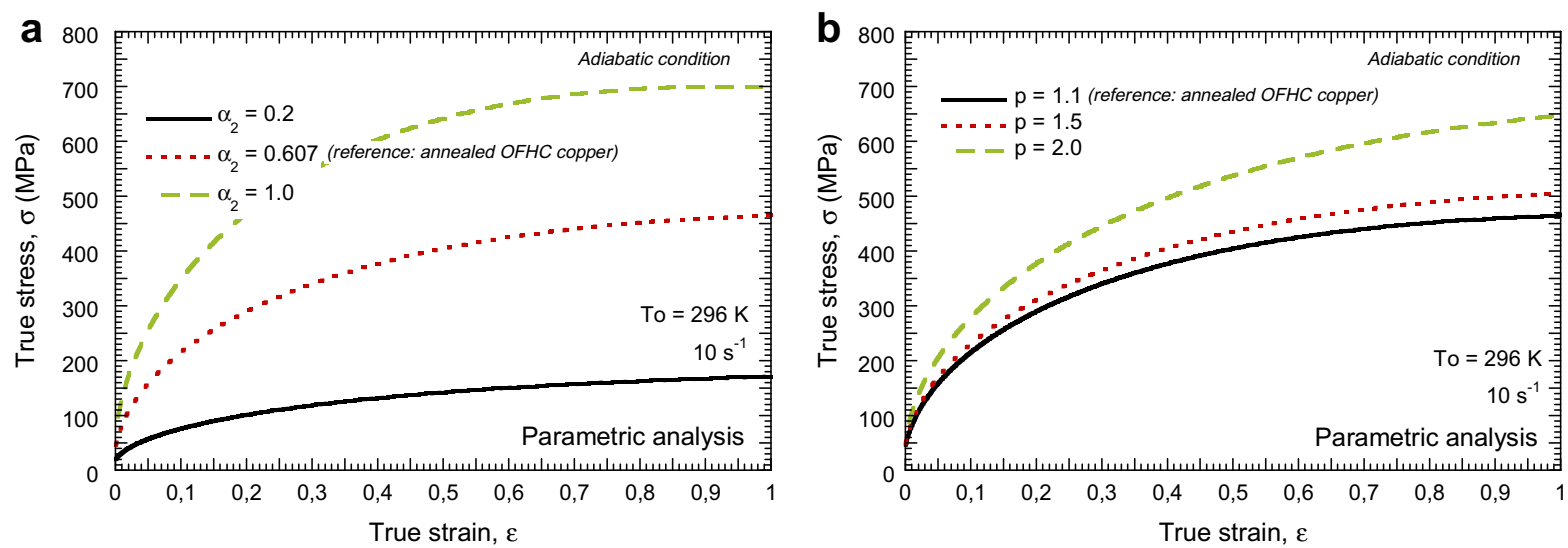

Fig. 5. Flow stress as a function of strain for different values of the material parameters (a) $\alpha_{2}$ and (b) $p$ Loading conditions: $10 \mathrm{~s}{ }^{1}$ and $T_{0}=296 \mathrm{~K}$.
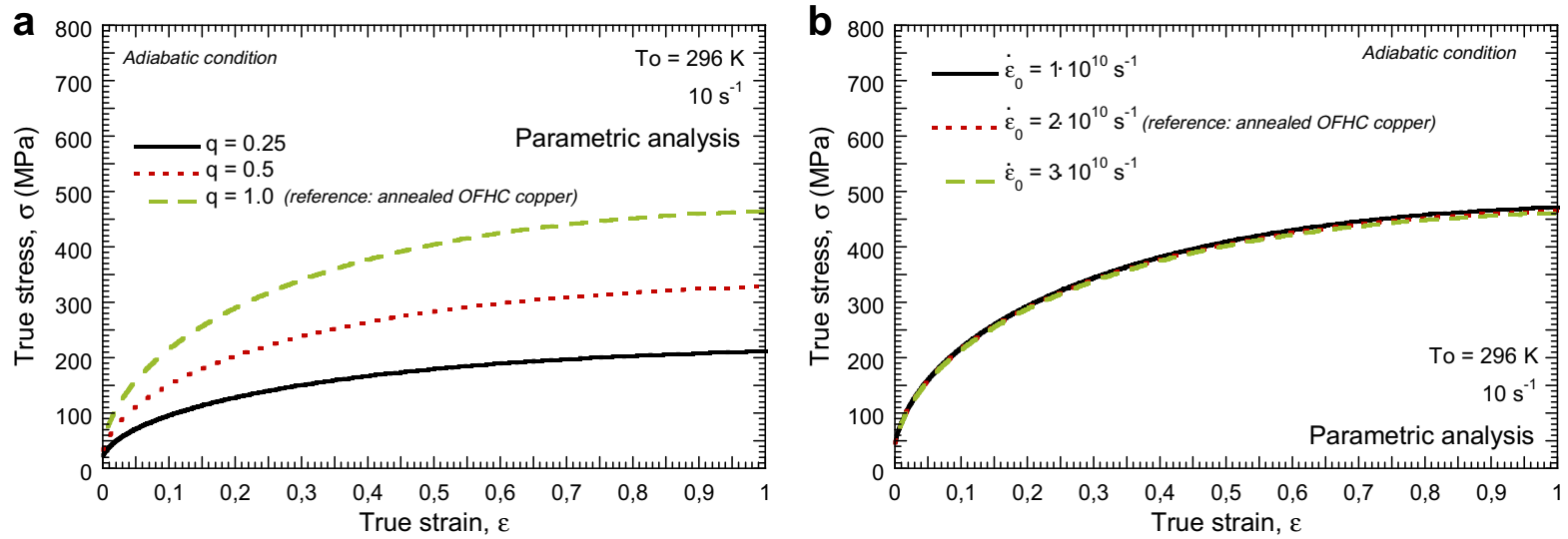

Fig. 6. Flow stress as a function of strain for different values of the material parameters (a) $q$ and (b) $\varepsilon_{0}$. Loading conditions: $10 \mathrm{~s}{ }^{1}$ and $T_{0}=296 \mathrm{~K}$.

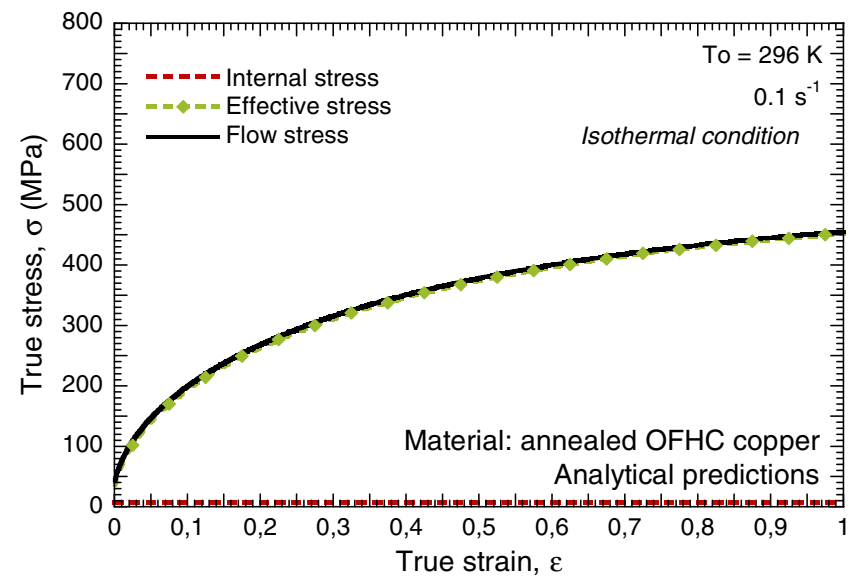

Fig. 7. Overall flow stress, internal stress and effective stress as a function of the plastic strain.

Fig. 10. Increasing rate sensitivity with plastic strain predicted by the constitutive description is in good corre lation with experimental results and theoretical consider ations for FCC metals reported for example in (Voyiadjis and Abed, 2005; Zerilli and Armstrong, 1987). This behav ior is caused by the evolution of the material structure with loading rate, which is gathered by the constitutive model. 

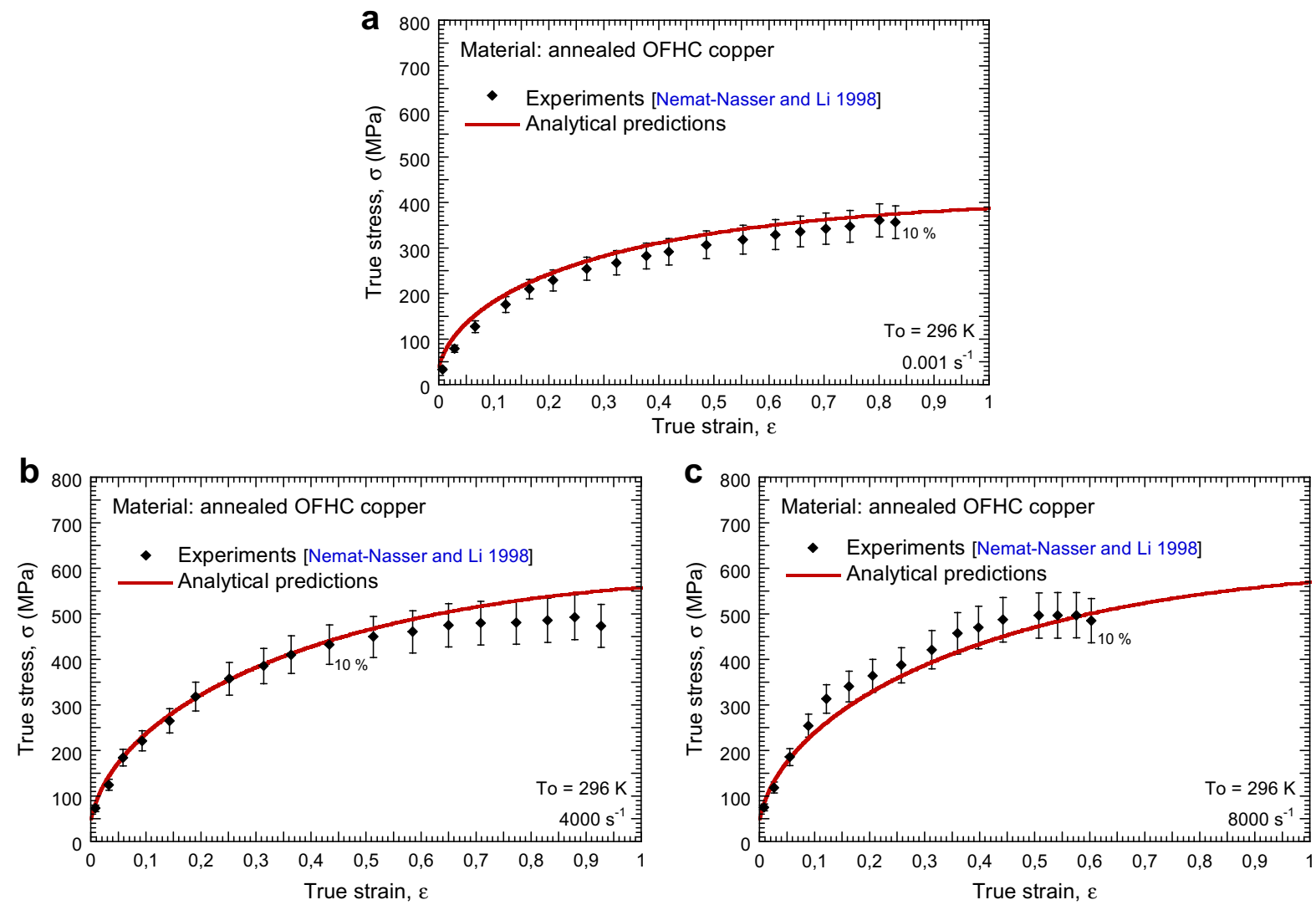

Fig. 8. Comparison between analytical predictions of the constitutive model and experiments at $T_{0}=296 \mathrm{~K}$ (Nemat-Nasser and Li, 1998 ). (a) $0.001 \mathrm{~s}{ }^{1}$, (b) $4000 \mathrm{~s}^{1}$., (c) $8000 \mathrm{~s}^{1}$.

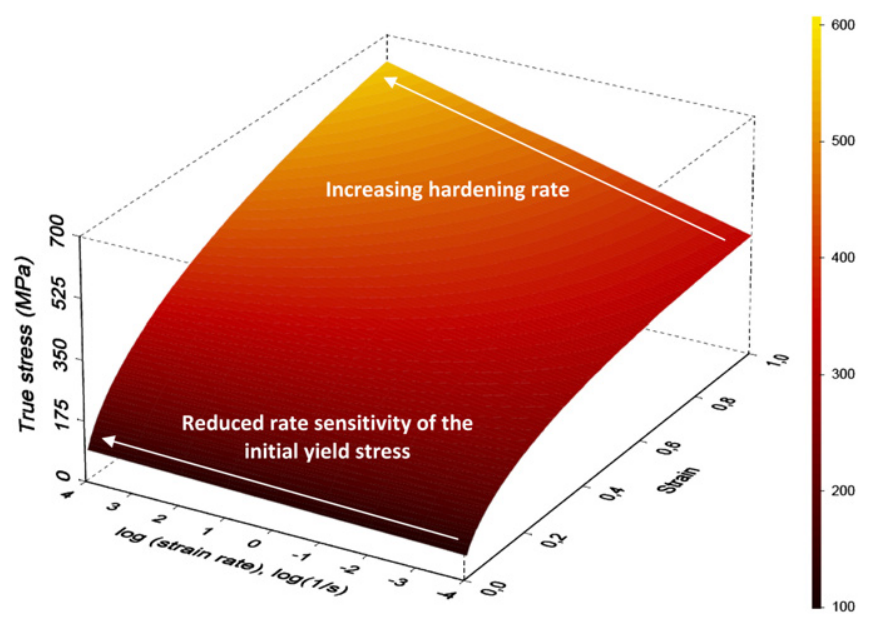

Fig. 9. Analytical predictions of the constitutive model within wide ranges of strain and strain rate at $T_{0}=296 \mathrm{~K}$. Material: annealed OFHC copper.

From low to moderate strains the constitutive relation describes properly the rate sensitivity (at constant strain) of the material, Fig. 10. Good correspondence between experiments and analytical predictions of the model is found at room temperature within wide ranges of strain rate. Moreover, it has to be noted that for the range $\dot{\bar{\varepsilon}}_{p}>8000 s{ }^{1}$ drag effects become relevant in the material behavior (Nemat Nasser and Li, 1998). Within that range of loading rates the model may underestimate the material rate sensitivity, Fig. 10. As previously mentioned, the max imum rate level for which this constitutive description shows applicability is $\dot{\bar{\varepsilon}}_{p}^{\max } \approx 8000 s^{1}$. 


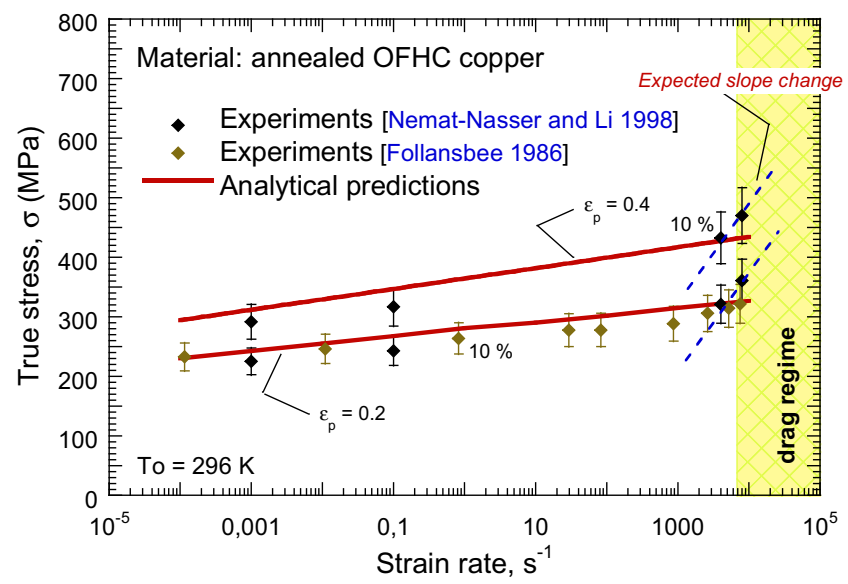

Fig. 10. Comparison between analytical predictions of the constitutive model and experiments within wide ranges of strain rate at different plastic strains and $T_{0}=296 \mathrm{~K}$ (Follansbee ,1986; Nemat-Nasser and Li, 1998).
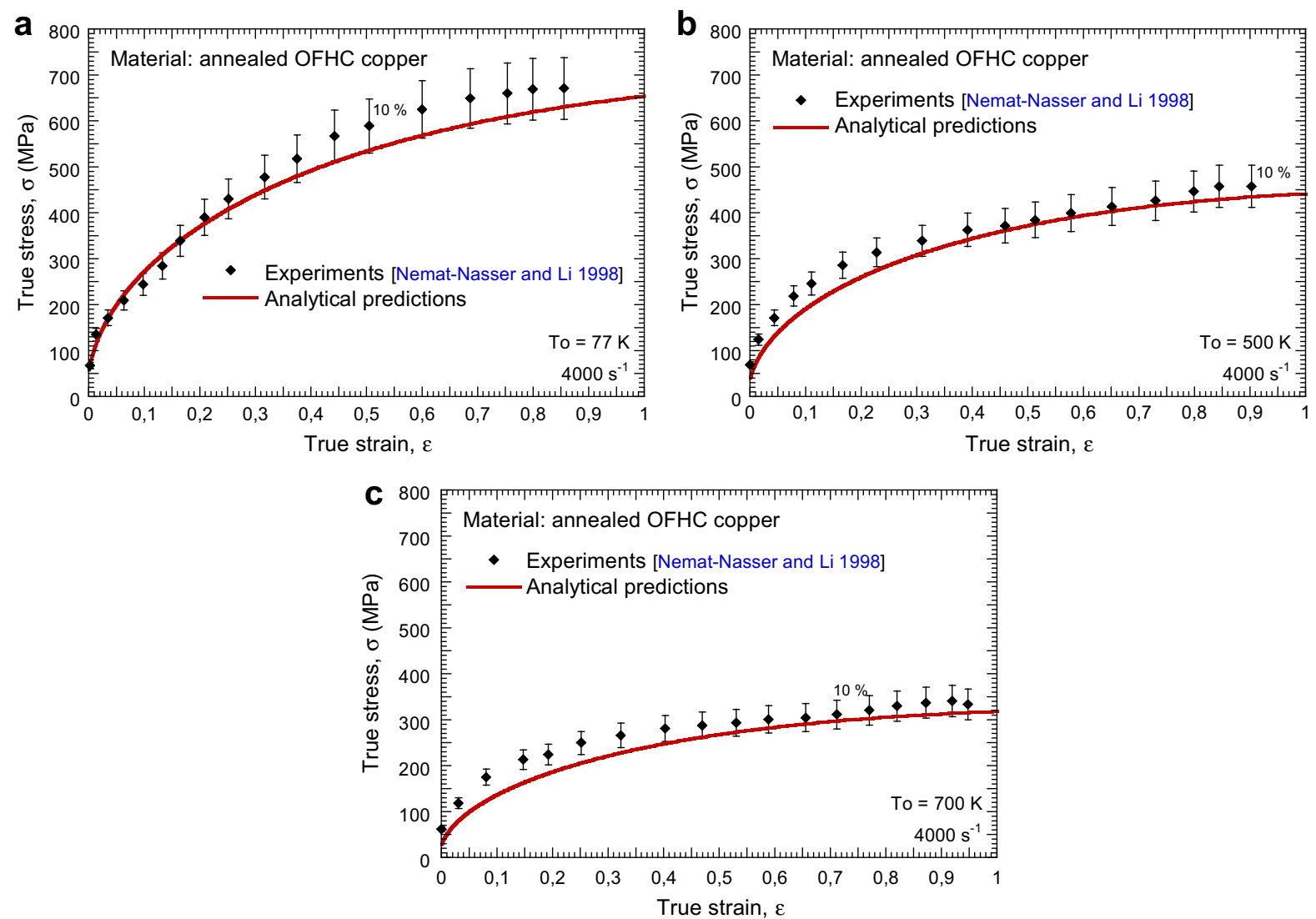

Fig. 11. Comparison between analytical predictions of the constitutive model and experiments at $4000 \mathrm{~s}{ }^{1}$ (Nemat-Nasser and Li, 1998 ). (a) $T_{0}=77 \mathrm{~K}$, (b) $T_{0}=500 \mathrm{~K},(\mathrm{c}) T_{0}=700 \mathrm{~K}$.

Moreover, in Fig. 11, is illustrated the agreement be tween experiments and analytical predictions in terms of stress strain curves for different initial temperatures at high loading rate. The constitutive model defines satis factorily the work hardening and the material flow stress within wide ranges of temperature, $77 \mathrm{~K}<T_{0}<700 \mathrm{~K}$. It was highlighted that strain rate influences material strain hardening, in the same manner it has to be re marked that temperature strongly influences work hard ening too. 


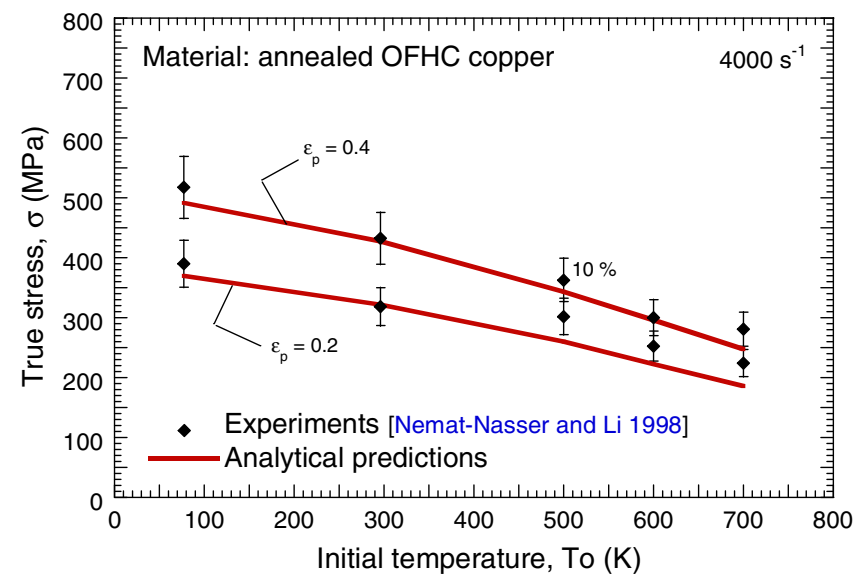

Fig. 12. Comparison between analytical predictions of the constitutive model and experiments within wide ranges of temperature at different plastic strains and $4000 \mathrm{~s}^{1}$ (Nemat-Nasser and Li, 1998).

Therefore, the material temperature sensitivity be comes strain dependent. This behavior is well defined by the model predictions, Fig. 12. According to experiments, analytical curves illustrating the evolution of the material flow stress as a function of temperature for different strain levels are not parallel, Fig. 12. Good correlation between experiments and model predictions is observed.

Thus, the constitutive description proposed is revealed suitable for describing the thermo viscoplastic response of the material within wide ranges of strain, strain rate and temperature. Especially relevant results the ability of the model to describe the distinctive dependence that strain hardening of annealed OFHC copper has on temper ature and loading rate.

Finally, it has to be noted that the one dimensional form of the constitutive relation developed in this paper may be easily generalized to three dimensional states of stress and strain following the procedure for integration of Huber Mises plasticity equations reported in (Rusinek et al., 2010; Zaera and Fernández Sáez, 2006).

\section{Conclusions and remarks}

In this paper a constitutive description with application to FCC metals is developed. The formulation is based on the decomposition of the material flow stress into internal stress and effective stress. The internal stress formulation takes into account the grain size effect; it is defined strain independent and shows athermal character. The effective stress formulation, which is the main innovative feature of this work, is founded on the concept of thermal activa tion analysis and takes into account the interrelationship between strain rate and temperature. It is structure depen dent via description of the dislocation density evolution as a function of plastic deformation. Dislocation density acts as internal state variable in the material deformation behavior. This provides information on the evolution of the material structure within wide ranges of strain rate and temperature.

Moreover, a systematic procedure for identification of the material parameters has been proposed. The analytical predictions of the constitutive description are compared with experimental data for annealed OFHC copper re ported in the literature. The constitutive model highlights due to its ability to describe the strain, rate and tempera ture dependences of the material flow stress.

\section{Acknowledgements}

The researchers of the University Carlos III of Madrid are indebted to the Comunidad Autónoma de Madrid (Pro ject CCG10 UC3M/DPI 5596) and to the Ministerio de Ciencia e Innovación de España (Project DPI/2008 06408) for the financial support received which allowed conduct ing part of this work.

\section{References}

Austin, R.A., McDowell, D.L., 2011. A dislocation-based constitutive model for viscoplastic deformation of FCC metals at very high strain rates. International Journal of Plasticity 27, 1-24.

Basinski, Z.S., 1959. Thermally activated glide in face-centred cubic metals and its application to the theory of strain hardening. Philosophical Magazine 4, 393-432.

Cai, M.C., Niu, L.S., Ma, X.F., Shi, H.J., 2010. A constitutive description of the strain rate and temperature effects on the mechanical behavior of materials. Mechanics of Materials 42, 774-781.

Campagne, L., Daridon, L., Ahzi, S., 2005. A physically based model for dynamic failure in ductile metals. Mechanics of Materials 37, 8:869886.

Campbell, J.D., 1973. Dynamic plasticity: macroscopic and microscopic aspects. Materials Science and Engineering 12 (1), 3-21.

Campbell, J.D., Ferguson, W.G., 1970. The temperature and strain-rate dependence of the shear strength of mild steel. Philosophical Magazine 81, 63-82.

Campbell, J.D., Harding, J., 1961. Response of metals to high velocity deformation. Interscience, New York and London, 51-74.

Conrad, H., 1961. On the mechanism of yielding and flow in iron. Journal of Iron and Steel Research International 198, 364.

Dolinski, M., Rittel, D., Dorogoy, A., 2010. Modeling adiabatic shear failure from energy considerations. Journal of the Mechanics and Physics of Solids 58, 1759-1775.

Estrin, Y., 1998. Dislocation theory based constitutive modelling: foundations and applications. Journal of Materials Processing Technology, 80-81,33-39.

Follansbee, P.S. 1986. High-strain-rate deformation of FCC metals and alloys. In: Metallurgical applications of shock-wave and high-strainrate phenomena. $451-479$. 
Gao, C.Y., Zhang, L.C., 2010. A constitutive model for dynamic plasticity of FCC metals. Materials Science and Engineering A 527, 3138-3143.

Huang, M., Rivera-Díaz-del-Castillo, P.E.J., Bouaziz, O., Van der Zwaag, S. 2009. A constitutive model for high-strain-rate deformation in FCC metals based on irreversible thermodynamics. Mechanics of Materials 41, 982-988.

Johnston, W.G., Gilman, J.J., 1959. Dislocation velocities, dislocation densities and plastic flow in lithium floride crystals. Journal of Applied Physics 30, 129-144.

Klepaczko, J.R., 1975. Thermally activated flow and strain rate history effects for some polycrystalline FCC metals. Material Science and Engineering 18, 121-135.

Klepaczko, J.R., Duffy, J., 1982. History effects in polycrystalline BCC metals and steel subjected to rapid changes in strain rate and temperature. Archives of Mechanics 34, 419-436.

Klepaczko, J.R., 1991. Physical-state variables - the key to constitutive modeling in dynamic plasticity. Nuclear Engineering and Design 127, $1: 103-115$.

Klepaczko, J.R., Rezaig, B., 1996. A numerical study of adiabatic shear bending in mild steel by dislocation mechanics based constitutive relations. Mechanics of Materials 24, 125-139.

Klepaczko, J.R. 1998. A general approach to rate sensitivity and constitutive modeling of FCC and BCC metals, in: Impact: Effects of Fast Transient Loadings, Rotterdam, 3-35.

Klepaczko, J.R., Rusinek, A., Rodríguez-Martínez, J.A., Pecherski, R.B., Arias, A., 2009. Modeling of thermo-viscoplastic behavior of $\mathrm{DH}-36$ and Weldox 460-E structural steels at wide ranges of strain rates and temperatures, comparison of constitutive relations for impact problems. Mechanics of Materials 41, 599-621.

Kocks, U.F., Argon, A.S., Ashby, M.F., 1975. Thermodynamics and kinetics of slip. In: Chalmers, B., Christian, J.W., Massalski, T.B. (Eds.), Progress in Materials Science, vol. 19. Oxford, Pergamon Press.

Kocks, U.F., 2001. Realistic constitutive relations for metal plasticity. Materials Science and Engineering A 317 (1-2), 181-187.

Kocks, U.F., Mecking, H., 2003. Physics and phenomenology of strain hardening: the FCC case. Progress in Materials Science 48, 171-273.

Kubin, L.P., Estrin, Y., 1990. Evolution of dislocation densities and the critical conditions for the Portevin-Le Châtelier effect. Acta Metallurgica Et Materialia 38 (5), 697-708.

Kumar, A., Hauser, F.E., Dorn, J.E., 1968. Viscous drag on dislocations in aluminum at high strain rates. Acta Metallurgica 9, 1189-1197.

Lennon, A.M., Ramesh, K.T., 2004. The influence of crystal structure on the dynamic behavior of materials at high temperatures. International Journal of Plasticity 20, 269-290.

Mecking, H., Kocks, U.F., 1981. Kinetics of flow and strain hardening. Acta Metallurgica 29, 1865-1875.

Meyers, M.A., Gregori, F., Kad, B.K., Schneider, M.S., Kalantar, D.H. Remington, B.A., Ravichandran, G., Boehly, T., Wark, J.S., 2003. Laserinduced shock compression of monocrystalline copper: characterization and analysis. Acta Materialia 51, 1211-1228.

Molinari, A., Ravichandran, G., 2005. Constitutive modeling of high-strainrate deformation in metals based on the evolution of an effective microstructural length. Mechanics of Materials 37, 737-752.

Nemat-Nasser, S., Li, Y., 1998. Flow stress of FCC polycrystals with application to OFHC Copper. Acta Materialia 46, 565-577.

Nemat-Nasser, S., Guo, W.G., 2003. Thermomechanical response of DH-36 structural steel over a wide range of strain rates and temperatures. Mechanics of Materials 35, 1023-1047.

Orowan, E., 1948. Discussion in Symposium on internal stresses in metals and alloys. Institute of Metals, London, 451.
Oussouaddi, O., Klepaczko, J.R., 1991. An analysis of transition from isothermal to adiabatic deformation in the case of a tube under torsion. Journal of Physics IV; Coll. C3 (Suppl.III), C3-323 [in French].

Perzyna, P., 1966. Fundamental problems in viscoplasticity, vol. 9. Academic Press, New York, pp. 243-377.

Rittel, D., Wang, Z.G., Merzer, M., 2006. Adiabatic shear failure and dynamic stored energy of cold work. Physical Review Letters 96, 075502.

Rusinek, A., Klepaczko, J.R., 2001. Shear testing of sheet steel at wide range of strain rates and a constitutive relation with strain-rate and temperature dependence of the flow stress. International Journal of Plasticity 17, 87-115.

Rusinek, A., Zaera, R., Klepaczko, J.R., 2007. Constitutive relations in 3-D for a wide range of strain rates and temperatures-application to mild steels. International Journal of Solids Structures 44, 5611-5634.

Rusinek, A., Rodríguez-Martínez, J.A., Klepaczko, J.R., Pecherski, R.B., 2009. Analysis of thermo-visco-plastic behavior of six high strength steels. Journal of Materials Des. 30, 1748-1761.

Rusinek, A., Rodríguez-Martínez, J.A., Arias, A., 2010. A thermoviscoplastic constitutive model for FCC metals with application to OFHC copper. International Journal of Mechanics Science 52 (2), 120135.

Seeger, A., 1957. The mechanism of glide and work-hardening in face centered cubic and hexagonal closed-packed metal. In: Dislocations and mechanical properties of crystal. Wiley., New York.

Tanimura, S., Duffy, J., 1986. Strain rate effects and temperature history effects for three different tempers of 4340 VAR steel. International Journal of Plasticity 2, 21-35.

Tanner, A.B., McDowell, D., 1999. Deformation, temperature and strain rate sequence experiments on $\mathrm{OFHC} \mathrm{Cu}$. International Journal of Plasticity 15 (4), 375-399.

Taylor, G.I., 1938. Plastic strain in metals. Journal of Inst. Metals 62, 307324.

Taylor, G., 1992. Thermally-activated deformation of BCC metals and alloys. Progress in Materials Science 36, 29-61.

Uenishi, A., Teodosiu, C., 2004. Constitutive modelling of the high strain rate behavior of interstitial-free steel. International Journal of Plasticity 20, 915-936.

Underwood, E.E., 1970. Quantitative Stereology. Addison-Wesley.

Voyiadjis, G.Z., Abed, F.H., 2005. Effect of dislocation density evolution on the thermomechanical response of metals with different crystal structures at low and high strain rates and temperatures. Archives of Mechanics 57 (4), 299-343.

Voyiadjis, G.Z., Almasri, A.H., 2008. A physically based constitutive model for FCC metals with applications to dynamic hardness. Mechanics of Materials 40, 549-563.

Zaera, R., Arias, A., Navarro, C., 2002. Analytical modelling of metallic circular plates subjected to impulsive loads. International Journal of Solids Structure 39, 659-672.

Zaera, R., Fernández-Sáez, J., 2006. An implicit consistent algorithm for the integration of thermoviscoplastic constitutive equations in adiabatic conditions and finite deformations. International Journal of Solids Structure 43, 1594-1612.

Zerilli, F.J., Armstrong, R.W., 1987. Dislocation-mechanics-based constitutive relations for material dynamics calculations. Journal of Applied Physics 61, 1816-1825.

Zerilli, F.J., Armstrong, R.W., 1992. The effect of dislocation drag on the stress-strain behavior of FCC metals. Acta Metallurgica Et Materialia 40, 1803-1808. 